\title{
Mst1 and Mst2 kinases: regulations and diseases
}

\author{
Funiu Qin ${ }^{\dagger}$, Jing $\operatorname{Tian}^{\dagger}$, Dawang Zhou ${ }^{*}$ and Lanfen Chen ${ }^{*}$
}

\begin{abstract}
The Hippo signaling pathway has emerged as a critical regulator for organ size control. The serine/threonine protein kinases Mst1 and Mst2, mammalian homologs of the Hippo kinase from Drosophila, play the central roles in the Hippo pathway controlling the cell proliferation, differentiation, and apoptosis during development. Mst1/2 can be activated by cellular stressors and the activation of Mst1/2 might enforce a feedback stimulation system to regulate oxidant levels through several mechanisms, in which regulation of cellular redox state might represent a tumor suppressor function of Mst1/2. As in Drosophila, murine Mst1/Mst2, in a redundant manner, negatively regulate the Yorkie ortholog YAP in multiple organs, although considerable diversification in the pathway composition and regulation is observed in some of them. Generally, loss of both Mst1 and Mst2 results in hyperproliferation and tumorigenesis that can be largely negated by the reduction or elimination of YAP. The Hippo pathway integrates with other signaling pathways e.g. Wnt and Notch pathways and coordinates with them to impact on the tumor pathogenesis and development. Furthermore, Mst1/2 kinases also act as an important regulator in immune cell activation, adhesion, migration, growth, and apoptosis. This review will focus on the recent updates on those aspects for the roles of Mst1/2 kinases.
\end{abstract}

Keywords: The Hippo pathway, Mst1, Mst2, Reactive oxygen species, YAP, Cancer, Immune diseases

\section{Introduction}

The Hippo pathway plays a very important role in controlling cell proliferation and differentiation, and monitoring organ size and oncogenesis. This pathway was first discovered in Drosophila through genetic screens for regulators of organ size. The loss of function (LOF) mutant of the protein kinase "Hippo" exhibits tissues overgrowth and tumorigenesis, in which the increased cell number is associated with the acceleration of cell cycle progression and a failure of developmental apoptosis [1-5]. The Hippo phenotype closely resembles phenotypes of LOF mutants of the protein kinase Warts $[6,7]$ and the small noncatlytic protein Mats [8] as well as a milder phenotype of another noncatalytic scaffold protein Salvador (Sav) $[9,10]$. Sav binds both Hippo and Warts, and promotes Hippo phosphorylation of Warts; Mats is another Hippo substrate that binds to and promotes Warts activation. With the activation of those downstream elements, the key role of Hippo signaling is to inhibit Yorkie $[11,12]$, a transcriptional coactivator of

\footnotetext{
* Correspondence: dwzhou@xmu.edu.cn; chenlanfen@xmu.edu.cn ${ }^{\dagger}$ Equal contributors

State Key Laboratory of Stress Cell Biology, School of Life Sciences, Xiamen University, Xiang'An South Road, Xiang'An District, Xiamen, Fujian 361102, China
}

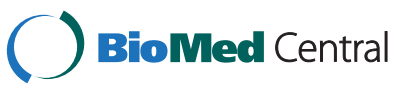

(c) 2013 Qin et al.; licensee BioMed Central Ltd. This is an Open Access article distributed under the terms of the Creative Commons Attribution License (http://creativecommons.org/licenses/by/2.0), which permits unrestricted use, distribution, and reproduction in any medium, provided the original work is properly cited. proliferative and pro-survival genes. These studies in Drosophila defined a developmentally regulated growthsuppressive and proapoptotic pathway operated by the Hippo kinase. Each of the core components of this pathway is evolutionally conserved and their counterpart(s) are identified in mammalians respectively. In general, the mammalian Ste20-like kinases Mst1 and Mst2 $[13,14]$ (Mst1/2, corresponding in Drosophila as Hippo), associated with the WW-domain scaffolding protein WW45 (corresponding in Drosophila as Sav), that binds Mst1/2 and phosphorylates Large tumor suppressor (Lats1/2, corresponding in Drosophila as Warts) [15], through their respective SARAH coiled coil domains, thereby promoting Mst1/2 phosphorylation of Lats; Mst1/2 also phosphorylates Mps one binder kinase activator-like 1 (Mob1A/B, corresponding in Drosophila as Mats) $[16,17]$ which enhances Mob1's ability to bind and activate Lats1/2; phospho-Mats binds to and promotes Wts/Lats autophosphorylation and activation; Lats1/2 phosphorylates Yes-associated protein (YAP, corresponding in Drosophila as Yki) [18], which promotes 14-3-3 binding to YAP, causing YAP nuclear exit, hereby inhibiting its function. Intranuclear YAP/Yki mainly promotes cell proliferation and resists cell death 
through the Scalloped/TEAD transcription factor(s). Loss of Mst1/Mst2 results in a YAP dependent accelerated proliferation, resistance to apoptosis and massive organ overgrowth. The details of many aspects of the Hippo signaling pathway can be found in depth discussion from several recent reviews [19-24]. In this review, we will focus on the recent updates of the roles of mammalian "Hippo" kinases, ie. Mst1 and Mst2, on the cellular redox state regulation and their involvement in organ size control, tumorigenesis, and immune regulation.

\section{Mst1/2 and the cellular redox state}

Oxidative stress induces the activation of Mst1/2 [25]. Thioredoxin-1 (Trx1), a conserved antioxidant protein that is well known for its disulfide reductase activity, can physically associate with the SARAH domain of Mst1 in intact cells and inhibit the homodimerization and autophosphorylation of Mst1, thereby prevents Mst1 activation; whereas $\mathrm{H} 2 \mathrm{O} 2$ abolishes this interaction and eventually causes the activation of Mst1. Thus, Trx-1 might function as a molecular switch to turn off the oxidative stress-induced activation of Mst1 [26]. Besides the Trx-1 as a redox-sensitive inhibitor of Mst1, the molecular mechanism of reactive oxygen species (ROS)-induced Mst1 activation needs to be further defined. Hippo/ Mst1 kinase directly phosphorylates and activates the forkhead box proteins (FOXO), which causes expression of proapoptotic genes, such as the FASL and TRAIL genes under stress conditions. The apoptosis of cultured neurons induced by oxidative stress or by Mst1 over expression is blocked by RNAi depletion of FOXO [27]. Mst1 mediates oxidative stress-induced neuronal cell death by phosphorylating the transcription factor FOXO3 at serine 207 [27], or FOXO1 at serine 212 [28]. Mst1 and its scaffold protein Nore1 are required in cell death of granule neurons upon growth factors deprivation and neuronal activity [28]. Yuan's group further demonstrates that oxidative stress induces the c-Abl-dependent tyrosine phosphorylation of Mst1 and increases the interaction between Mst1 and FOXO3, thereby activating the Mst1-FOXO signaling pathway, leading to cell death in both primary culture neurons and rat hippocampal neurons. These results suggest that c-Abl-Mst-FOXO signaling cascade plays an important role in cellular responses to oxidative stress and might contribute to pathological states including neurodegenerative diseases in the mammalian central nerve system (CNS) [29,30]. Indeed, Mst1 mediated FoxO3 activation in response to $\beta$-amyloid $(\mathrm{A} \beta)$ has been shown to mediate death of selective neuron in Alzheimer's disease (AD) [31]. Furthermore, amyotrophic lateral sclerosis (ALS) associated SOD1(G93A) mutant induces dissociation of Mat1 from a redox protein trx-1 and promotes Mst1 activation in spinal cord neurons in a reactive oxygen species-dependent manner. Genetic deficiency of Mst1 delays disease onset and extends survival in mice expressing the ALSassociated G93A mutant of human SOD1 [32].

Lim's group recently also shows that Hippo-Foxa2 signaling pathway plays a role in peripheral lung maturation and surfactant homeostasis [33]. In the immune system, Mst1 deficient peripheral T cells have impaired FOXO1/3 and decreased FOXO protein levels indicating a crucial role of the Mst1-FOXO signaling pathway for the maintenance of naive $\mathrm{T}$ cell homeostasis [34]. Mst1 deficient lymphocytes and neutrophils exhibit enhanced loss of mitochondrial membrane potential and increased susceptibility to apoptosis [35]. More recently, Valis K. et al. further demonstrated that the activation of Hippo/Mst1 is able to stimulate the transcription of another proapoptotic mediator NOXA in a FOXO1-dependent Manner via acetylation of the histone proteins in the NOXA promoter [36]. The Hippo/Mst1-FOXO1-Noxa axis is a novel tumor suppressor pathway that controls apoptosis in cancer cells exposed to anticancer drugs such as a-TOS [36]. In contrast, a recent study demonstrates that Ras activation and mitochondrial dysfunction cooperatively stimulate production of ROS resulting in activation of JNK signaling which cooperates with oncogenic Ras to inactivate the Hippo pathway, leading to up regulation of YAP targets Unpaired (an Interleukin-6 homologue) and Wingless (a Wnt homologue) in Drosophila [37], although earlier study show activated K-Ras induces apoptosis by engaging the RASSF1A-Mst2-Lats1 pathway [38].

Recently, Morinaka et al. demonstrate that peroxiredoxin1 (Prdx1), a cysteine-containing, highly conserved enzyme that reduces $\mathrm{H} 2 \mathrm{O} 2$ to $\mathrm{H} 2 \mathrm{O}$ and $\mathrm{O} 2$, interacts with Mst1 under conditions of oxidative stress and Prdx1 is required for Mst1 activation by $\mathrm{H} 2 \mathrm{O} 2$, as knockdown of Prdx1 is associated with loss of Mst1 activity [39]. Chernoff's group also shows that both Mst1 and Mst2 interact with Prdx1 in HEK-293 or in human hepatocarcinoma HepG2 cells under oxidative stress conditions [40]. However, the later one supports that Prdx1 represents a downstream target, rather than an upstream regulator of Mst1. Mst1 phosphorylates Prdx1 at the highly conserved Thr-183 site resulting in inactivation of Prdx1 with subsequent increased $\mathrm{H} 2 \mathrm{O} 2$ levels in cells. As Mst1 can be activated by increased $\mathrm{H} 2 \mathrm{O} 2$ levels, inactivation of Prdx1 resulted from the activated Mst1 might enforce a feedback stimulation system to prolong or intensify Mst1 activation. Such a feedback stimulation system, resulting in higher oxidant levels and DNA damage, might represent a tumor suppressor function of Mst1/2 to prevent the accumulation of mutations [40]. Consistently, our recent study shows that elimination of Mst1/2 from liver cells is accompanied by increased expression of a cohort of anti-oxidant enzymes important for ROS elimination 
[41]. The increased expression levels of those enzymes, such as glutathione reductase (GSR), NAD(P)H:quinone oxidoreductase (NQO1), $\gamma$-glutamyl-cysteine ligase (GCL, including catalytic subunit (GCLC) and modifier subunit (GCLM)), catalase (CAT), copper/zinc superoxide dismutase (SOD), cytosolic thioredoxin (Txn1) and mitochondrial thioredoxin (Txn2), promote the accumulation of glutathione (GSH). The accumulation of GSH in the Mst1/ 2 deficient liver results in the activation of the GA-binding protein (GABP) which is a critical transcription factor for the expression of YAP [41,42]. In addition, Mst2-Lats1 can physically bind and promotes phosphorylation of GABP $\beta$ which interrupts $\mathrm{GABP} \alpha / \beta$ homodimerization, prevents their nuclear localization and inhibits their transcriptional activity. Thus, in addition to inhibit YAP function by phosphorylation of YAP and promoting YAP nuclear exit, Mst1/2-Lats signaling can also inhibit YAP function by downregulating its expression level [41]. In contrast to the Mst1-FOXO signaling pathway leading to the decreased ROS production, the activation of the Mst1/2 pathway inhibiting YAP in liver tissues maintains the higher levels of ROS (Figure 1). There is no doubt that oxidative stress activates Mst1/2 signaling; however the conflict effects on regulating the cellular oxidative state upon the activation of Mst1/2 are reported in different cell contexts. It is possible that the Mst-FOXO signaling pathway is predominantly activated in neuron or immune cells resulting in the decreased ROS production, whereas in other cell types, such as hepatocyte, the activation of Mst1/2-GABP-YAP signaling leads to increased ROS production. These critical but inconsistent findings indicate the importance and complexity of inter-regu lation among mitochondrial function, oxidant generation and/or clearance, and the Hippo signaling pathway.

Increased production of ROS during pro-oxidant conditions would lead to Mst1/2 activation resulting in phosphorylation of GABP, inhibition of its transcription activity, and downregulation of YAP expression, consequently decreased the expression of a variety of genes that encode mitochondrial proteins and proteins with antioxidant properties, resulting in increased cellular ROS and a diminished GSH/GSSG ratio [41]. On the other hand, GABP itself helps modulate oxidative metabolism of the cell through regulating the expression of many genes necessary for cellular respiration in mitochondria, including enzymes involved in oxidative phosphorylation, such as cytochrome c oxidase subunits IV and Vb [43]. Growing evidence points that the cellular redox state and redox signaling has significant roles in regulating the metabolic fate and regenerative potential of adult tissues $[44,45]$. The GABP will emerge as a critical component of the Hippo signaling pathway for its role in regulating the cellular redox state and cell growth.

\section{The roles of Mst1/2 in organ size control and tumorigenesis}

The Hippo signaling pathway is a tumor suppresser pathway. Mst1 or Mst2 single knockout mice are viable and do not exhibit obvious organ overgrowth or tumor development, whereas Mst1 and Mst2 double-knockout (DKO) mice exhibit early embryonic lethality $[46,47]$. To define the roles of Mst1 and Mst2 in vivo, conditional

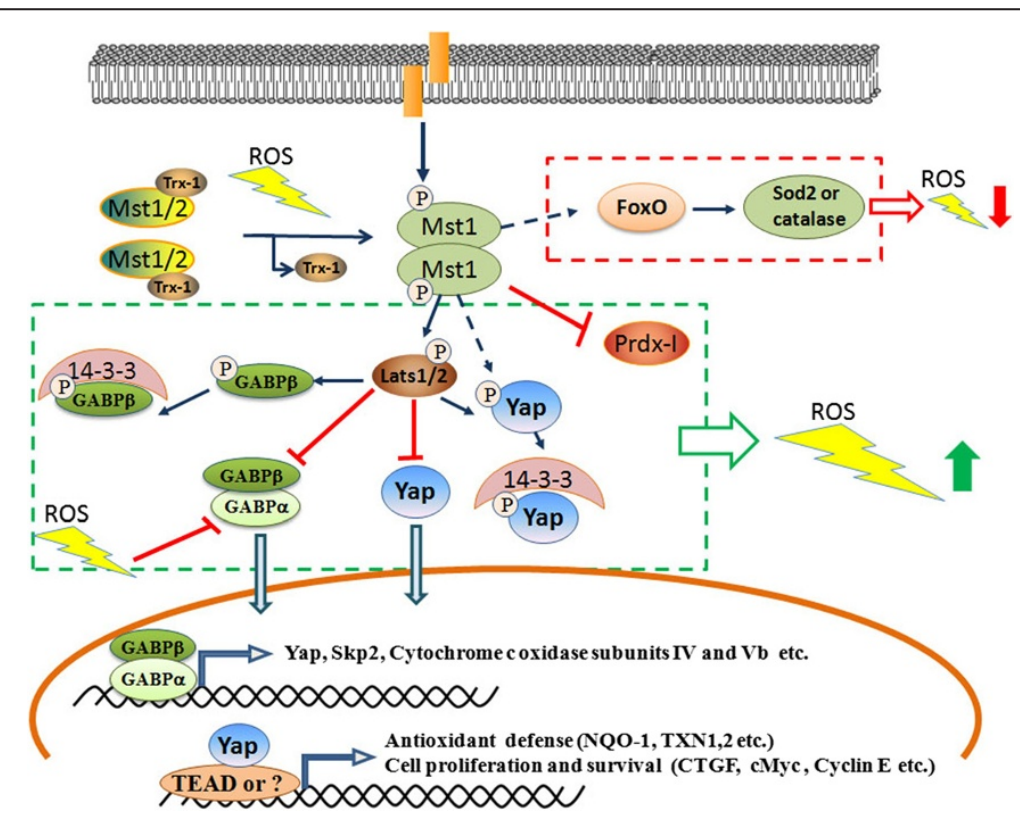

Figure 1 Mammalian Mst1 and Mst2 kinases play essential role in the regulation of cellular redox state. See text for details. 
knockout mouse of Mst1 and Mst2 in variety tissues were generated and severe context-dependent phenotypes were observed (Table 1). For example, Hippo seems to control cell-cycle exit and terminal differentiation in some tissues without having major effects on organ growth, whereas in other tissues Hippo signaling maintains stem cell/progenitor compartments. The Hippo-Lats-Yorkie tumor-suppressor pathway predicated in Drosophila does not prevail in all mammalian tissues. In mammalian liver, Mst1/Mst2 negatively regulates Yap1, whereas, in mouse embryo fibroblasts (MEFs), the cell-cell contact results in Yap1 phosphorylation and nuclear exclusion equally well in wild type and Mst1/Mst2 DKO MEFs [46]; in mouse keratinocytes, Yap inactivation during cellular differentiation occurs independently of Mst1/2 and lats1/2 [48]. Thus, it appears that the wiring upstream of Yap1 and downstream of Mst1/Mst2 has been diversified considerably in mammals compared with the Drosophila Hippo pathway.

\section{Liver}

We and other groups have demonstrated that Mst1 and Mst2 are the most potent tumor suppressors in liver and a single copy of either Mst1 or Mst2 can significantly inhibit tumor formation in the liver $[46,49,50]$. Elimination of both alleles of Mst1 together with heterozygosity for Mst2, and vice versa, results in the development of spontaneous hepatocellular carcinomas associated with loss of the remaining wild-type Mst1 or Mst2 allele in the tumors, whereas no tumors were observed in other organs of these mice. Conditional inactivation of Mst1/ Mst2 in the liver results in the immediate onset of dramatic hepatocyte proliferation and hepatomegaly followed by the development of Hepatocellular carcinoma (HCC) and cholangiocarcinoma within 2 month, in which loss of Mst1/2-dependent inhibition of YAP contributes to the liver cell proliferation and tumorigenesis.

Inactivation of Mst1/Mst2 in liver leads to the loss of YAP(Ser127) phosphorylation and increased YAP nuclear localization. Knocking-down YAP in Mst1/Mst2deficient HCC cell lines results in massive cell death and cell cycle arrest, similarly, the restoration of Mst1 expression in these cells restores YAP(Ser127) phosphorylation and leads to cell cycle arrest and apoptosis. In contrast to Drosophila, Lats1/2 does not serve as the Mst1/Mst2 activated YAP kinase in hepatocytes, indicating the existence of an novel, as yet unidentified intermediary kinase downstream of Mst1/Mst2 that is critical for YAP(Ser127) phosphorylation in the liver [46]. However, our recently study shows that activation of Mst2/ Lats1 can downregulate the expression of YAP by regulating GABP $\beta 1$ phosphorylation and cytoplasmic retention in HepG2 Cells. Besides reduced YAP(Ser127) phosphorylation, the relative expression levels of YAP have also been shown significantly increased in human HCCs compared with nontumorous livers [41]. Nevertheless, both the upstream regulation of Mst1/2 and the full spectrum of Mst1/2 antiproliferative targets remain to be defined as do the relative role of these pathways in promoting hepatic carcinogenesis [51].

\section{Intestines}

The intestines of Mst1 or Mst2 single knockout mice are indistinguishable from their wild-type counterparts. Mst1/2 intestinal DKO mice (Mst $\left.1^{-/} M s t 2^{f l / f l}-v i l l i n-C r e\right)$ with ablation of both Mst1 and Mst2 in intestinal compartment are born normal at birth, however they develop colonic adenomas within 3 months old and can only survive for about 13 weeks (median age) accompanied by severe wasting. Both the small and large intestine of $M s t 1^{-/-} M s t 2^{f l f l}$-villin-Cre mice exhibit an expansion of stem-like undifferentiated cells expressing high levels of CD133, Leucine-rich repeat-containing G-protein coupled receptor 5 (Lgr5) and Achaete-scute complex homolog 2 (Ascl2), which are stem cell markers in the intestine, an increased number of cells expressing CD44 and CD24, markers associated with colon cancer stem cells, and an almost complete absence of all secretory lineages. The loss of Mst1/2 in intestine decreases phosphorylation of YAP(Ser127 and Ser384) and causes an increase in both YAP abundance and nuclear localization. The hyperproliferation and loss of differentiation caused by the Mst1/2 deficiency can be entirely

Table 1 Phenotypes of the Mst1/2 conditional knockout mice

\begin{tabular}{|c|c|c|}
\hline Affected tissue & Phenotypes of Mst1/2 deficient mice & References \\
\hline Liver & $\begin{array}{l}\text { Dramatic hepatocyte proliferation and hepatomegaly; Development of hepatocellular carcinoma (HCC) and } \\
\text { cholangiocarcinoma within } 2 \text { months. }\end{array}$ & $46,49,50$ \\
\hline Intestines & $\begin{array}{l}\text { Intestinal dysplasia; An expansion of stem-like undifferentiated cells; An almost complete absence of all secretory } \\
\text { lineages; Development of the polypoid lesions and colonic adenomas within } 3 \text { months old. }\end{array}$ & 52 \\
\hline Pancreas & $\begin{array}{l}\text { A significantly decrease in pancreas mass; Acinar cell atrophy; Overabundance of ductal structures; Smaller islets with } \\
\text { abnormal } a / \beta \text { cell ratios in pancreas }\end{array}$ & 59,60 \\
\hline Heart & $\begin{array}{l}\text { Expansion of trabecular and subcompact ventricular myocardial layers; Thickened ventricular walls, and enlarged } \\
\text { ventricular chambers without a change in myocardial cell size. }\end{array}$ & 66 \\
\hline
\end{tabular}


reversed by deleting a single YAP allele in $\mathrm{Mst}^{-/-} \mathrm{Mst} 2^{\mathrm{fl} /}$

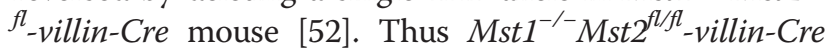
mouse exhibits similar phenotype to the transgenic mice overexpressing YAP(Ser127Ala) in the small intestinal compartment, wherein intestinal dysplasia and loss of goblet and Paneth cells are also observed [52,53].

The inactivation of Mst1/2 in the intestine compartment to promote the hyperproliferation of intestinal stem cells and to inhibit intestinal epithelial differentiation is attributed largely to an enhancement of $\beta$ catenin action and an activation of Notch signaling. The enhanced $\beta$-catenin transcriptional activity in the intestine compartment of $\mathrm{Mst}^{-/-} \mathrm{Mst2} 2^{\mathrm{fl} / \mathrm{fl}}$-villin-Cre mouse is evident by the increased abundance of the activated form of $\beta$-catenin (dephospho-Ser37/Thr41) and Wnt targets Lgr5 and Ascl2 [52]. The expression levels of the Notch ligand Jagged 1, mediated possibly in part through up-regulated Wnt signaling [54,55], the intranuclear Notch intracellular domain (NICD) and the abundance of Hairy and enhancer of split 1 (Hes1), a Notch target gene, are all increased in Mst1/Mst2 deficient intestine. Those evidences indicate that the Notch signaling pathway is highly activated in the intestine of $M s t 1^{-/-} M s t 2^{f l}$

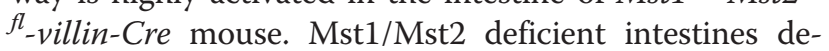
velop colonic adenomas, and unlike the polyps described in the Sav1-deficient colon [56], the polypoid lesions in the Mst1/Mst2-deficient colon do not exhibit a sawtooth/serrated architecture but hyperproliferative adenoma which might result from an activation of $\beta$-catenin and/or the inactivation of the Hippo signaling pathway in these lesions $[52,57]$.

\section{Pancreas}

The Hippo pathway is necessary for proper development and to preserve homeostasis in the liver and intestines, both of which, as well as the pancreas, are developed from a primitive gut tube derived from the embryonic endoderm [58]. Thus the pancreas specific Mst1 and Mst2 conditional knockout mice using Pdx1-Cre were generated to study the effect of the Hippo pathway during mouse pancreas development. Mst1/2 pancreasspecific knockout (Mst1/2-Pdx-Cre) mice were born with no distinctive pancreatic defects at birth, however, in contrast to Mst1/2 liver-specific knockout mice with the hepatomegaly phenotype, Mst1/2-Pdx-Cre mice have a significantly decrease in pancreas mass relative to that of wild-type littermate controls at adult age [59,60]. These mice exhibit obvious morphologic alterations, including acinar cell atrophy, overabundance of ductal structures, and smaller islets with abnormal $\alpha / \beta$ cell ratios in pancreas. In brief, the pancreas became more ductal and less acinar in phenotype. Furthermore, a YAP-dependent loss of acinar cell identity and extensive disorganization in Mst1/2 deficient exocrine tissue leads to pancreatitis-like autodigestion which might result in tissue necrosis and pancreas mass decrease.

In mouse embryo, normal pancreatic differentiation is divided to two stages, the primary transition and the secondary transition. The primary transition occurring between embryonic days 9.5 and 12.5 (E9.5 and E12.5 respectively) marks the appearance of very low levels of acinar digestive enzymes and the first wave glucagon-gene and subsequently insulin-gene expressing cells. The secondary transition (between E13.5 and E16.5) characterized by intense proliferation and differentiation throughout the pancreas epithelium spans the geometric increase of acinar digestive enzymes and insulin [61]. Mst1 (but not Mst2) and YAP proteins are detected in the wild type pancreas during the secondary transition stage, and was almost undetectable at birth before returning to higher levels at postnatal day 7 (P7) and P14. Mst1/2 deficiency does not affect YAP protein levels in the embryonic pancreas, but lost of Mst1/2 was associated with higher levels of total YAP at adult age [59]. Within the adult pancreas, Yap expression is limited to the exocrine compartment, including ductal and acinar cells, whereas loss of Mst1/2 increases the YAP protein level and nuclear accumulation of nearly all exocrine cells accompanied with increased cell proliferation rate. Those evidences suggested that Mst $1 / 2$ signaling does not play a major role in pancreas organogenesis, but become functionally active during the secondary transition. The activation of Mst1/2 is required for regulating postnatal YAP levels and phosphorylation status in acinar cells to maintain differentiation $[59,60]$.

\section{Heart}

It has been shown that Mst1 regulates heart size by activating its downstream kinase, Lats2, and inhibiting YAP activity, thereby attenuating compensatory cardiomyocyte growth. In cardiomyocytes, Mst1 is activated by pathological stimuli, such as hypoxia/reoxygenation in vitro and ischemia/reperfusion in vivo [62]. Mst1 mediates cardiac troponin I phosphorylation and play a critical role in the modulation of myofilament function in the heart. The function of Mst1 in cardiomyocytes can also be negatively regulated by a new identified Mst1-interacting protein protein-L-isoaspartate (D-aspartate) O-methyltransferase (PCMT1) [63]. Cardiac-specific over-expression of Mst1 in mouse results in activation of caspases, increased apoptosis and dilated cardiomyopathy, whereas the inhibition of endogenous Mst1 prevents apoptosis of cardiomyocytes and cardiac dysfunction after myocardial infarction without producing cardiac hypertrophy [62,64]. Furthermore, Del Re DP and colleagues show that Rassf1A is an endogenous activator of Mst1 in the heart and the function of Rassf1A/Mst1 pathway is different between cardiomyocytes and fibroblasts. The Rassf1A/Mst1 pathway promotes apoptosis in cardiomyocytes playing a detrimental 
role; while the same pathway inhibits fibroblast proliferation and cardiac hypertrophy through both cellautonomous and autocrine/paracrine mechanisms, playing a protective role during pressure overload [65]. More recently, cardiac conditional knockout mice with either WW45, Lats2 or Mst1/2 using the Nkx2.5-cre exhibit expansion of trabecular and subcompact ventricular myocardial layers, thickened ventricular walls, and enlarged ventricular chambers without a change in myocardial cell size [66]. Yap1 protein was robustly detected in neonatal and juvenile mouse heart and declined with age. Cardiomyocyte-restricted loss of Yap1 in Fetal resulted in marked, lethal myocardial hypoplasia and decreased cardiomyocyte proliferation, whereas fetal activation of Yap1 stimulated cardiomyocyte proliferation [67]. Thus, the Mst1/2-WW45/Lats2-Yap1 pathway is critical of cardiomyocyte proliferation, cardiac morphogenesis, and myocardial trabeculation, but it does not influence physiological hypertrophic growth of cardiomyocytes during the experimental context. Gene expression profiling and chromatin immunoprecipitation revealed that Hippo signaling negatively regulates a subset of Wnt target gene in cardiomyocyte [66].

\section{The functions of Mst $1 / 2$ in immune system}

The murine Mst1 and Mst2 kinases are most abundant in tissues of the lymphoid system. Mst1 kinase acts as an important regulator in $\mathrm{T}$ cell selection, adhesion, migration, growth, and apoptosis [68-73]. The Mst1 deficient mouse exhibits a reduction in white pulp, decreased numbers of total $\mathrm{CD}^{+}{ }^{+} \mathrm{T}$ cells, $\mathrm{CD} 8^{+} \mathrm{T}$ cells and $\mathrm{B} 220^{+} \mathrm{B}$ cells and absence of marginal zone $\mathrm{B}$ cells. Compared to the wild type littermates, Mst1-deficient mice have much fewer CD $62 \mathrm{~L}^{\text {hi }} / \mathrm{CD} 44^{\text {lo }}$ naïve peripheral $\mathrm{T}$ cells and a high proportion of CD62 $\mathrm{L}^{\mathrm{lo}} / \mathrm{CD} 44^{\mathrm{hi}}$ effector/memory T cells in tissues, such as liver and lung. Inactivation of Mst1 and Mst2 does not have obvious effect on the thymocytes development, although a lightly small size thymus is found in the Mst1 ${ }^{-/} M s t 2^{f l / f l}$-VavCre mouse. This might due to the very low abundance and activity of Mst1/2 kinases in double-positive (DP) cells and developmentally earlier thymocytes. Recently, patients bearing LOF mutations of Mst1 are reported with a primary immunodeficiency syndrome characterized by naïve $\mathrm{CD}^{+}$and $\mathrm{CD} 8^{+} \mathrm{T}$-cell lymphopenia in particular, as well as neutropenia, closely assembling with the major defect of Mst1 deficient mice in lymphocyte homeostasis. Those patients have recurrent bacterial infections, viral infections, and autoimmune manifestations with autoantibodies [35,74,75]. In contrast to defects seen with deletion of Mst1, a global deletion of Mst2 caused no changes in lymphocyte numbers in any compartment. However, the additional elimination of Mst2 in the entire hematopoietic lineage on an Mst1 defi-

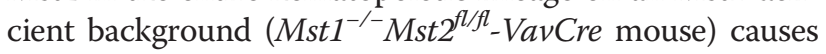

a marked exacerbation of the deficits seen in Mst1 deficient $\mathrm{T}$ cells, suggesting that Mst2 might play a redundant role in lymphoid tissues during the absence of Mst1 [69]. The kinase activity of Mst1 is essence for T cell homeostasis, since the defective phenotype of Mst1/Mst2 deficiency in the lymphoid compartment can only be restored by the transgenic expression of wild type but not catalytically inactive Mst1.

Mst1-deficient naive $\mathrm{T}$ cells proliferate vigorously in response to TCR stimulation and have enhanced ongoing apoptosis in vivo. Mst1, but not Mst2, is greatly reduced in effector/memory $\mathrm{T}$ cells compared to that in naive $\mathrm{T}$ cells, thus Mst1 might serves as a likely determinant of the threshold for activation of naïve $\mathrm{T}$ cells. Upon the $\mathrm{T}$ cell receptor (TCR) stimulation, the increase in tyrosine phosphorylation of CD3 , ZAP70, Lck, and PLCY is similar in splenic $\mathrm{T}$ cells from wild-type and Mst1 deficient mice, whereas the phosphorylation of Mob1A/B observed in the wild-type T cells is lost entirely in the Mst1 deficient T cells. Elimination of Mst1 has little effect on the Lats1 carboxyl-terminal phosphorylation, Lats1/2 autophosphorylation and YAP phosphorylation in T cells. Thus the activation of Mob1A/B might serve as the effector of Mst1's antiproliferative effect in naïve $T$ cells $[69,71]$. The disruption of Mst1, or both Mst1 and Mst2, impairs the thymocyte egress and causes an accumulation of nature T cells in thymus, shown as the increased proportion of singlepositive (SP) thymocytes in thymus, and a decreased number of lymphocytes in circulation. Mst1-deficient mice show defects in adhesion, homing, and intranodal migration in vivo. Furthermore, two independent pools of the ADAP/SKAP55 module, one of which associates with RAPL, Mst1, and Rap1, whereas the other interacts with RIAM, Mst1, Kindlin-3, and Talin are identified that they are independently recruited to the $\alpha$ - or $\beta$-chain of LFA-1 and coordinate CCR7-mediated activation of LFA1 as well as T-cell adhesion and migration [76]. Thymocytes express multiple Rac1/2 GEFs [77], in which the deletion of Dock2 resulting in similar defects in migration, actin polarization, and Rac GTPase activation seen in the Rac1/Rac2-deficient thymocytes [78]. Mst1/Mst2 double knockout thymocytes lack the ability to activate RhoA as well as Rac, however, no evidence shows that Dock2 is a regulated downstream of Mst1/Mst2. Although the limited overlap between Dock8 and Mst1/Mst2 deficiency, loss of phospho-Mob1A/B activation of Dock8 might contribute to chemokine-stimulated Rac1 activation in Mst1/Mst2-deficient thymocytes and in turn to the failure of thymic egress [69]. More recently, Mst1 in thymocyte has also been shown to involve in LFA-1/ ICAM-1-dependent high-velocity medullary migration and is required for migrating thymocytes to associate with rare populations of Aire ${ }^{+}$ICAM- ${ }^{\text {hi }}$ mTECs in a negatively selecting environment. Thus, Mst1 might 
have a key role in regulating thymocyte self-antigen scanning in the medulla [79].

\section{Conclusion}

The mammalian Hippo pathway has generated great interests and gained significant progress in the past few years. In addition to the conserved role of growth control and tumor prevention, the Hippo pathway has also been shown to integrate with other critical signaling pathways, such as Wnt and Notch pathways and extend its function in many other critical biological events. There are still many open questions in the Hippo pathway field remained to be fully elucidated, especially the mechanism by which upstream regulators of the Hippo pathway to initiate or terminate signaling, and how the cellular redox plays a role in this process. Advances in understanding the Hippo signaling pathway regulation may not only solve the scientific questions, such as organ size control and developmental regulations, but also provide new therapeutic targets for human diseases.

\section{Abbreviations}

DKO: Double-knockout; FoxO: Forkhead box protein; GABP: GA-binding protein; GSH: Glutathione; Lats1/2: Large tumor suppressor; LOF: Loss of function; MEFs: Mouse embryo fibroblasts; Mob1A/B: Mps one binder kinase activator-like 1; Mst1/2: Mammalian Ste20-like kinases; Prdx1: Peroxiredoxin-1; ROS: Reactive oxygen species; TCR: T cell receptor; Trx1: Thioredoxin-1; WW45: WW-domain scaffolding protein; YAP: Yes-associated protein.
\end{abstract}

\section{Competing interests}

The authors declare that they have no competing interests.

\section{Authors' contributions}

All authors drafted and approved the manuscript.

\begin{abstract}
Acknowledgments
This work was supported by grants from the China's 1000 Young Talents Program (to D.Z.), the 111 Project of the Ministration of Education of China (B06016), the Fundamental Research Funds for the Central Universities of China (2010111079), the National Natural Science Foundation of China (81101503/H1602, 31270918/C080101, and 81222030/H1602), the Natural Science Foundation of Fujian (2011 J05096), and the Program of Introducing Talents of Discipline to Universities (B12001).
\end{abstract}

Received: 21 June 2013 Accepted: 3 August 2013

Published: 28 August 2013

\section{References}

1. Wu S, Huang J, Dong J, Pan D: Hippo encodes a Ste-20 family protein kinase that restricts cell proliferation and promotes apoptosis in conjunction with salvador and warts. Cell 2003, 114(4):445-456.

2. Harvey KF, Pfleger CM, Hariharan IK: The Drosophila Mst ortholog, hippo, restricts growth and cell proliferation and promotes apoptosis. Cell 2003, 114(4):457-467.

3. Udan RS, Kango-Singh M, Nolo R, Tao C, Halder G: Hippo promotes proliferation arrest and apoptosis in the Salvador/Warts pathway. Nat Cell Biol 2003, 5(10):914-920.

4. Pantalacci S, Tapon N, Leopold P: The Salvador partner Hippo promotes apoptosis and cell-cycle exit in Drosophila. Nat Cell Biol 2003, 5(10):921-927.

5. Jia J, Zhang W, Wang B, Trinko R, Jiang J: The Drosophila Ste20 family kinase dMST functions as a tumor suppressor by restricting cell proliferation and promoting apoptosis. Genes Dev 2003, 17(20):2514-2519.

6. Justice RW, Zilian O, Woods DF, Noll M, Bryant PJ: The Drosophila tumor suppressor gene warts encodes a homolog of human myotonic dystrophy kinase and is required for the control of cell shape and proliferation. Genes Dev 1995, 9(5):534-546.

7. Xu T, Wang W, Zhang S, Stewart RA, Yu W: Identifying tumor suppressors in genetic mosaics: the Drosophila lats gene encodes a putative protein kinase. Development 1995, 121(4):1053-1063.

8. Lai ZC, Wei X, Shimizu T, Ramos E, Rohrbaugh M, Nikolaidis N, Ho LL, Li Y: Control of cell proliferation and apoptosis by mob as tumor suppressor, mats. Cell 2005, 120(5):675-685.

9. Tapon N, Harvey KF, Bell DW, Wahrer DC, Schiripo TA, Haber DA, Hariharan IK: salvador Promotes both cell cycle exit and apoptosis in Drosophila and is mutated in human cancer cell lines. Cell 2002, 110(4):467-478.

10. Kango-Singh M, Nolo R, Tao C, Verstreken P, Hiesinger PR, Bellen HJ, Halder $\mathrm{G}$ : Shar-pei mediates cell proliferation arrest during imaginal disc growth in Drosophila. Development 2002, 129(24):5719-5730.

11. Huang J, Wu S, Barrera J, Matthews K, Pan D: The Hippo signaling pathway coordinately regulates cell proliferation and apoptosis by inactivating Yorkie, the Drosophila Homolog of YAP. Cell 2005, 122(3):421-434.

12. Dong J, Feldmann G, Huang J, Wu S, Zhang N, Comerford SA, Gayyed MF, Anders RA, Maitra A, Pan D: Elucidation of a universal size-control mechanism in Drosophila and mammals. Cell 2007, 130(6):1120-1133.

13. Creasy CL, Ambrose DM, Chernoff J: The Ste20-like protein kinase, Mst1, dimerizes and contains an inhibitory domain. J Biol Chem 1996, 271(35):21049-21053.

14. Creasy $\mathrm{CL}$, Chernoff J: Cloning and characterization of a member of the MST subfamily of Ste20-like kinases. Gene 1995, 167(1-2):303-306.

15. Chan EH, Nousiainen M, Chalamalasetty RB, Schafer A, Nigg EA, Sillje HH: The Ste20-like kinase Mst2 activates the human large tumor suppressor kinase Lats1. Oncogene 2005, 24(12):2076-2086.

16. Praskova M, Xia F, Avruch J: MOBKL1A/MOBKL1B phosphorylation by MST1 and MST2 inhibits cell proliferation. Curr Biol 2008, 18(5):311-321.

17. Wei $X$, Shimizu T, Lai ZC: Mob as tumor suppressor is activated by Hippo kinase for growth inhibition in Drosophila. EMBO J 2007, 26(7):1772-1781.

18. Zhao B, Wei X, Li W, Udan RS, Yang Q, Kim J, Xie J, Ikenoue T, Yu J, Li L, et al: Inactivation of YAP oncoprotein by the Hippo pathway is involved in cell contact inhibition and tissue growth control. Genes Dev 2007, 21(21):2747-2761.

19. Sudol M, Shields DC, Farooq A: Structures of YAP protein domains reveal promising targets for development of new cancer drugs. Semin Cell Dev Biol 2012, 23(7):827-833.

20. Avruch J, Zhou D, Fitamant J, Bardeesy N, Mou F, Barrufet LR: Protein kinases of the Hippo pathway: regulation and substrates. Semin Cell Dev Biol 2012, 23(7):770-784.

21. Hong W, Guan KL: The YAP and TAZ transcription co-activators: key downstream effectors of the mammalian Hippo pathway. Semin Cell Dev Biol 2012, 23(7):785-793.

22. Chen L, Qin F, Deng X, Avruch J, Zhou D: Hippo pathway in intestinal homeostasis and tumorigenesis. Protein \& cell 2012, 3(4):305-310.

23. Zhao B, Tumaneng K, Guan KL: The Hippo pathway in organ size control, tissue regeneration and stem cell self-renewal. Nat Cell Biol 2011, 13(8):877-883.

24. Radu M, Chernoff J: The DeMSTification of mammalian Ste20 kinases. Curr Biol 2009, 19(10):R421-R425.

25. Graves JD, Gotoh Y, Draves KE, Ambrose D, Han DK, Wright M, Chernoff J, Clark EA, Krebs EG: Caspase-mediated activation and induction of apoptosis by the mammalian Ste20-like kinase Mst1. EMBO J 1998, 17(8):2224-2234

26. Chae JS, Gil Hwang S, Lim DS, Choi EJ: Thioredoxin-1 functions as a molecular switch regulating the oxidative stress-induced activation of MST1. Free Radic Biol Med 2012, 53(12):2335-2343.

27. Lehtinen MK, Yuan Z, Boag PR, Yang Y, Villen J, Becker EB, DiBacco S, de la Iglesia N, Gygi S, Blackwell TK, et al: A conserved MST-FOXO signaling pathway mediates oxidative-stress responses and extends life span. Cell 2006, 125(5):987-1001.

28. Yuan Z, Lehtinen MK, Merlo P, Villen J, Gygi S, Bonni A: Regulation of neuronal cell death by MST1-FOXO1 signaling. J Biol Chem 2009, 284(17):11285-11292.

29. Xiao L, Chen D, Hu P, Wu J, Liu W, Zhao Y, Cao M, Fang Y, Bi W, Zheng Z, et al: The c-Abl-MST1 signaling pathway mediates oxidative stress-induced neuronal cell death. J Neurosci Off J Soc Neurosci 2011, 31(26):9611-9619.

30. Liu W, Wu J, Xiao L, Bai Y, Qu A, Zheng Z, Yuan Z: Regulation of neuronal cell death by c-Abl-Hippo/MST2 signaling pathway. PloS one 2012, 7(5):e36562. 
31. Sanphui P, Biswas SC: FoxO3a is activated and executes neuron death via Bim in response to beta-amyloid. Cell Death Dis 2013, 4:e625.

32. Lee JK, Shin JH, Hwang SG, Gwag BJ, McKee AC, Lee J, Kowall NW, Ryu H, Lim DS, Choi EJ: MST1 functions as a key modulator of neurodegeneration in a mouse model of ALS. Proc Natl Acad Sci U S A 2013, 110(29):12066-12071.

33. Chung C, Kim T, Kim M, Song H, Kim TS, Seo E, Lee SH, Kim H, Kim SK, Yoo $G$, et al: Hippo-Foxa2 signaling pathway plays a role in peripheral lung maturation and surfactant homeostasis. Proc Natl Acad Sci U S A 2013, 110(19):7732-7737.

34. Choi J, Oh S, Lee D, Oh HJ, Park JY, Lee SB, Lim DS: Mst1-FoxO signaling protects Naive $T$ lymphocytes from cellular oxidative stress in mice. Plos one 2009, 4(11):e8011.

35. Abdollahpour H, Appaswamy G, Kotlarz D, Diestelhorst J, Beier R, Schaffer AA, Gertz EM, Schambach A, Kreipe HH, Pfeifer D, et al: The phenotype of human STK4 deficiency. Blood 2012, 119(15):3450-3457.

36. Valis K, Prochazka L, Boura E, Chladova J, Obsil T, Rohlena J, Truksa J, Dong LF, Ralph SJ, Neuzil J: Hippo/Mst1 stimulates transcription of the proapoptotic mediator NOXA in a FoxO1-dependent manner. Cancer Res 2011, 71(3):946-954.

37. Ohsawa $S$, Sato $Y$, Enomoto $M$, Nakamura $M$, Betsumiya A, Igaki T: Mitochondrial defect drives non-autonomous tumour progression through Hippo signalling in Drosophila. Nature 2012, 490(7421):547-551.

38. Matallanas D, Romano D, Al-Mulla F, O'Neill E, Al-Ali W, Crespo P, Doyle B, Nixon C, Sansom O, Drosten M, et al: Mutant K-Ras activation of the proapoptotic MST2 pathway is antagonized by wild-type K-Ras. Mol Cell 2011, 44(6):893-906

39. Morinaka A, Funato $Y$, Uesugi $K$, Miki H: Oligomeric peroxiredoxin-I is an essential intermediate for p53 to activate MST1 kinase and apoptosis. Oncogene 2011, 30(40):4208-4218.

40. Rawat SJ, Creasy CL, Peterson JR, Chernoff J: The tumor suppressor Mst1 promotes changes in the cellular redox state by phosphorylation and inactivation of peroxiredoxin-1 protein. J Biol Chem 2013, 288(12):8762-8771.

41. Wu H, Xiao Y, Zhang S, Ji S, Wei L, Fan F, Geng J, Tian J, Sun X, Qin F, et al: The Ets transcription factor GABP is a component of the hippo pathway essential for growth and antioxidant defense. Cell reports 2013, 3(5):1663-1677.

42. Chinenov $Y$, Schmidt T, Yang XY, Martin ME: Identification of redox-sensitive cysteines in GA-binding protein-alpha that regulate DNA binding and heterodimerization. J Biol Chem 1998, 273(11):6203-6209.

43. Rosmarin AG, Resendes KK, Yang Z, McMillan JN, Fleming SL: GA-binding protein transcription factor: a review of GABP as an integrator of intracellular signaling and protein-protein interactions. Blood Cells $\mathrm{Mol}$ Dis 2004, 32(1):143-154.

44. Circu ML, Aw TY: Intestinal redox biology and oxidative stress. Semin Cell Dev Biol 2012, 23(7):729-737.

45. Zhang $\mathrm{H}$, Forman $\mathrm{HJ}$ : Glutathione synthesis and its role in redox signaling. Semin Cell Dev Biol 2012, 23(7):722-728.

46. Zhou D, Conrad C, Xia F, Park JS, Payer B, Yin Y, Lauwers GY, Thasler W, Lee JT, Avruch J, et al: Mst1 and Mst2 maintain hepatocyte quiescence and suppress hepatocellular carcinoma development through inactivation of the Yap1 oncogene. Cancer Cell 2009, 16(5):425-438.

47. Oh S, Lee D, Kim T, Kim TS, Oh HJ, Hwang CY, Kong YY, Kwon KS, Lim DS: Crucial role for Mst1 and Mst2 kinases in early embryonic development of the mouse. Mol Cell Biol 2009, 29(23):6309-6320.

48. Schlegelmilch K, Mohseni M, Kirak O, Pruszak J, Rodriguez JR, Zhou D, Kreger BT, Vasioukhin V, Avruch J, Brummelkamp TR, et al: Yap1 acts downstream of alpha-catenin to control epidermal proliferation. Cell 2011, 144(5):782-795.

49. Lu L, Li Y, Kim SM, Bossuyt W, Liu P, Qiu Q, Wang Y, Halder G, Finegold MJ, Lee JS, et al: Hippo signaling is a potent in vivo growth and tumor suppressor pathway in the mammalian liver. Proc Natl Acad Sci U S A 2010, 107(4):1437-1442.

50. Song H, Mak KK, Topol L, Yun K, Hu J, Garrett L, Chen Y, Park O, Chang J, Simpson RM, et al: Mammalian Mst1 and Mst2 kinases play essential roles in organ size control and tumor suppression. Proc Natl Acad Sci U S A 2010, 107(4):1431-1436.

51. Lee KP, Lee JH, Kim TS, Kim TH, Park HD, Byun JS, Kim MC, Jeong WI, Calvisi DF, Kim JM, et al: The Hippo-Salvador pathway restrains hepatic oval cell proliferation, liver size, and liver tumorigenesis. Proc Natl Acad Sci U S A 2011, 107(18):8248-8253.
52. Zhou D, Zhang $Y$, Wu H, Barry E, Yin $Y$, Lawrence $E$, Dawson D, Willis JE, Markowitz SD, Camargo FD, et al: Mst1 and Mst2 protein kinases restrain intestinal stem cell proliferation and colonic tumorigenesis by inhibition of Yes-associated protein (Yap) overabundance. Proc Natl Acad Sci U S A 2011, 108(49):E1312-E1320.

53. Camargo FD, Gokhale $S$, Johnnidis JB, Fu D, Bell GW, Jaenisch R, Brummelkamp TR: YAP1 increases organ size and expands undifferentiated progenitor cells. Curr Biol 2007, 17(23):2054-2060.

54. Pannequin J, Bonnans C, Delaunay N, Ryan J, Bourgaux JF, Joubert D, Hollande F: The wnt target jagged-1 mediates the activation of notch signaling by progastrin in human colorectal cancer cells. Cancer Res 2009, 69(15):6065-6073.

55. Rodilla V, Villanueva A, Obrador-Hevia A, Robert-Moreno A, FernandezMajada V, Grilli A, Lopez-Bigas N, Bellora N, Alba MM, Torres F, et al: Jagged1 is the pathological link between Wnt and Notch pathways in colorectal cancer. Proc Natl Acad Sci U S A 2009, 106(15):6315-6320.

56. Cai J, Zhang N, Zheng Y, de Wilde RF, Maitra A, Pan D: The Hippo signaling pathway restricts the oncogenic potential of an intestinal regeneration program. Genes Dev 2010, 24(21):2383-2388.

57. Rosenbluh J, Nijhawan D, Cox AG, Li X, Neal JT, Schafer EJ, Zack TI, Wang X, Tsherniak A, Schinzel AC, et al: beta-Catenin-driven cancers require a YAP1 transcriptional complex for survival and tumorigenesis. Cell 2012, 151(7):1457-1473.

58. Spence JR, Lauf R, Shroyer NF: Vertebrate intestinal endoderm development. Dev Dyn 2011, 240(3):501-520.

59. Gao T, Zhou D, Yang C, Singh T, Penzo-Mendez A, Maddipati R, Tzatsos A, Bardeesy N, Avruch J, Stanger BZ: Hippo signaling regulates differentiation and maintenance in the exocrine pancreas. Gastroenterology 2013 144(7):1543-1553. e1541.

60. George NM, Day CE, Boerner BP, Johnson RL, Sarvetnick NE: Hippo signaling regulates pancreas development through inactivation of Yap. Mol Cell Biol 2012, 32(24):5116-5128.

61. Stanger BZ, Hebrok M: Control of cell identity in pancreas development and regeneration. Gastroenterology 2013, 144(6):1170-1179.

62. Yamamoto S, Yang G, Zablocki D, Liu J, Hong C, Kim S-J, Soler S, Odashima $M$, Thaisz J, Yehia $G$, et al: Activation of Mst1 causes dilated cardiomyopathy by stimulating apoptosis without compensatory ventricular myocyte hypertrophy. J Clin Invest 2003, 111(10):1463-1474.

63. Yan G, Qin Q, Yi B, Chuprun K, Sun H, Huang S, Sun J: Protein-Lisoaspartate (D-aspartate) O-methyltransferase protects cardiomyocytes against hypoxia induced apoptosis through inhibiting proapoptotic kinase Mst1. Int J Cardiol 2013, S0167-5273(13):00629-00633.

64. Odashima M, Usui S, Takagi H, Hong C, Liu J, Yokota M, Sadoshima J: Inhibition of endogenous Mst1 prevents apoptosis and cardiac dysfunction without affecting cardiac hypertrophy after myocardial infarction. Circ Res 2007, 100(9):1344-1352.

65. Del Re DP, Matsuda T, Zhai P, Gao S, Clark GJ, Van Der Weyden L, Sadoshima J: Proapoptotic Rassf1A/Mst1 signaling in cardiac fibroblasts is protective against pressure overload in mice. J Clin Invest 2010, 120(10):3555-3567.

66. Heallen T, Zhang M, Wang J, Bonilla-Claudio M, Klysik E, Johnson RL, Martin JF: Hippo pathway inhibits Wnt signaling to restrain cardiomyocyte proliferation and heart size. Science 2011, 332(6028):458-461.

67. von Gise A, Lin Z, Schlegelmilch K, Honor LB, Pan GM, Buck JN, Ma Q, Ishiwata T, Zhou B, Camargo FD, et al: YAP1, the nuclear target of Hippo signaling, stimulates heart growth through cardiomyocyte proliferation but not hypertrophy. Proc Natl Acad Sci U S A 2012, 109(7):2394-2399.

68. Dong $Y$, Du X, Ye J, Han M, Xu T, Zhuang Y, Tao W: A cell-intrinsic role for Mst1 in regulating thymocyte egress. J Immunol 2009, 183(6):3865-3872.

69. Mou F, Praskova M, Xia F, van Buren D, Hock H, Avruch J, Zhou D: The Mst1 and Mst2 kinases control activation of rho family GTPases and thymic egress of mature thymocytes. J Exp Med 2012, 209(4):741-759.

70. Katagiri K, Imamura M, Kinashi T: Spatiotemporal regulation of the kinase Mst1 by binding protein RAPL is critical for lymphocyte polarity and adhesion. Nat Immunol 2006, 7(9):919-928.

71. Zhou D, Medoff BD, Chen L, Li L, Zhang XF, Praskova M, Liu M, Landry A Blumberg RS, Boussiotis VA, et al: The Nore1B/Mst1 complex restrains antigen receptor-induced proliferation of naive T cells. Proc Natl Acad SCi U S A 2008, 105(51):20321-20326.

72. Katagiri K, Ueda Y, Tomiyama T, Yasuda K, Toda Y, Ikehara S, Nakayama Kl, Kinashi T: Deficiency of Rap1-binding protein RAPL causes 
lymphoproliferative disorders through mislocalization of p27kip1. Immunity 2011, 34(1):24-38.

73. Katagiri K, Katakai T, Ebisuno Y, Ueda Y, Okada T, Kinashi T: Mst1 controls lymphocyte trafficking and interstitial motility within lymph nodes. EMBO J 2009, 28(9):1319-1331.

74. Nehme NT, Pachlopnik Schmid J, Debeurme F, Andre-Schmutz I, Lim A, Nitschke P, Rieux-Laucat F, Lutz P, Picard C, Mahlaoui N, et al: MST1 mutations in autosomal recessive primary immunodeficiency characterized by defective naive T cells survival. Blood 2012, 119(15):3458-3468.

75. Crequer A, Picard C, Patin E, D'Amico A, Abhyankar A, Munzer M, Debre M, Zhang SY, de Saint-Basile G, Fischer A, et al: Inherited MST1 deficiency underlies susceptibility to EV-HPV infections. PloS one 2012, 7(8):e44010.

76. Kliche S, Worbs T, Wang X, Degen J, Patzak I, Meineke B, Togni M, Moser $M$, Reinhold A, Kiefer F, et al: CCR7-mediated LFA-1 functions in T cells are regulated by 2 independent ADAP/SKAP55 modules. Blood 2012, 119(3):777-785.

77. Tybulewicz VL, Henderson RB: Rho family GTPases and their regulators in lymphocytes. Nat Rev Immunol 2009, 9(9):630-644.

78. Faroudi M, Hons M, Zachacz A, Dumont C, Lyck R, Stein JV, Tybulewicz VL: Critical roles for Rac GTPases in T-cell migration to and within lymph nodes. Blood 2010, 116(25):5536-5547.

79. Ueda Y, Katagiri K, Tomiyama T, Yasuda K, Habiro K, Katakai T, Ikehara S, Matsumoto M, Kinashi T: Mst1 regulates integrin-dependent thymocyte trafficking and antigen recognition in the thymus. Nat Commun 2012, 3:1098.

doi:10.1186/2045-3701-3-31

Cite this article as: Qin et al:: Mst1 and Mst2 kinases: regulations and diseases. Cell \& Bioscience 2013 3:31.

\section{Submit your next manuscript to BioMed Central and take full advantage of:}

- Convenient online submission

- Thorough peer review

- No space constraints or color figure charges

- Immediate publication on acceptance

- Inclusion in PubMed, CAS, Scopus and Google Scholar

- Research which is freely available for redistribution 